\title{
A New Method to Estimate the Contribution of Geometrical Optics in Arbitrary Linear Stratified Planar Structures
}

\author{
Vito Daniele and Guido Lombardi
}

\begin{abstract}
Studying the contribution of geometrical optics in arbitrary linear stratified planar structures is of great importance in practical problems. In this article we propose a new procedure based on the Bresler-Marcuvitz transversalization method and equivalent network modeling that is useful for computing source contributions in Wiener-Hopf formulations of complex scattering problems where angular or stratified structures are present. The generalized Wiener-Hopf technique has demonstrated the capability to handle new complex canonical problems through both exact and semianalytical factorization methods.
\end{abstract}

\section{Introduction}

The Wiener-Hopf technique in its generalized form has been applied effectively in electromagnetic wave scattering problems for angular regions (wedge problems); see $[1,2]$ and references therein. Following the procedure first proposed in [3], we aim to extend the Wiener-Hopf technique in angular regions for arbitrary linear wave scattering problems [3-6]. This technique can be also extended to geometries containing angular regions or stratified planar regions; see, for instance, [7]. We start our formulation from electromagnetic applications [3-5] and extend the procedure to elasticity as reported in [6]. The method is based on two steps: the deduction of the generalized Wiener-Hopf equations for angular-region problems [3-6] and the solution of the equations using the semianalytical factorization procedure known as Fredholm factorization; see, for instance, $[8,9]$. A key point in implementing the solution of generalized Wiener-Hopf equations for arbitrary linear stratified media via Fredholm factorization is the extraction of the source term that is related to geometrical-optics components. For this reason, this article is dedicated to estimating the contribution of geometrical optics in arbitrary linear stratified planar structures with the help of equivalent network models and the Bresler-Marcuvitz transversalization method.

\section{Transverse Equations in Stratified Media}

In this article we use only time harmonic fields with a time dependence specified by the factor $e^{j \omega t}$,

Manuscript received 16 December 2021.

Vito Daniele and Guido Lombardi are with Politecnico di Torino, Corso Duca degli Abruzzi 24, 10129, Torino, Italy; e-mail: vito.daniele@polito.it, guido.lombardi@polito.it. This work was supported by the Italian Ministry of University and Research under PRIN grant no. 2017NT5W7Z GREEN TAGS which is omitted. In the absence of finite-located sources, Maxwell's equations assume the abstract form $[5,10]$ in the Euclidean space of dimension 6 :

$$
\left(\Gamma_{\nabla}-\mathrm{W}\right) \tilde{\psi}=0
$$

where

$$
\begin{gathered}
\tilde{\boldsymbol{\psi}}=|\tilde{\mathbf{E}}, \tilde{\mathbf{H}}|^{t}, \quad \Gamma_{\nabla}=\left|\begin{array}{cc}
0 & 1_{3} \times \nabla \\
1_{3} \times \nabla & 0
\end{array}\right|, \\
\mathrm{W}=j \omega\left|\begin{array}{cc}
\boldsymbol{\varepsilon} & \xi \\
-\zeta & -\boldsymbol{\mu}
\end{array}\right|
\end{gathered}
$$

with $\mathrm{W}$ containing the dyadic permittivity $\varepsilon$, the dyadic permeability $\boldsymbol{\mu}$, and the additional coupling parameters

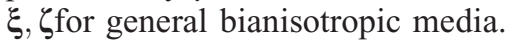

We introduce Cartesian coordinates $(z, x, y)$ and consider stratification along the $y$ direction.

The study of the wave motion in stratified media is significantly simplified if we introduce the transverse equations of the fields. These equations involve only the components $\tilde{\psi}_{t}$ of the field $\tilde{\psi}$ that remain continuous along the stratification according to the boundary conditions on the interfaces. According to the boundary conditions, we have

$$
\tilde{\boldsymbol{\psi}}_{t}=\left|\tilde{E}_{z}, \tilde{E}_{x}, 0, \tilde{H}_{z}, \tilde{H}_{x}, 0\right|^{t}=\mathbf{I}_{t} \cdot \tilde{\psi}
$$

where $\mathbf{I}_{t}=\operatorname{diag}[1,1,0,1,1,0]$. The transverse equations are obtained using [10] as reported in [5]:

$$
-\frac{\partial}{\partial y} \tilde{\psi}_{t}=\tilde{M}\left(\frac{\partial}{\partial z}, \frac{\partial}{\partial x}\right) \cdot \tilde{\psi}_{t}
$$

with matrix differential operator

$$
\begin{aligned}
& \tilde{\mathbf{M}}\left(\frac{\partial}{\partial z}, \frac{\partial}{\partial x}\right) \\
& \quad=-\Gamma_{y}\left[\left(W_{t y}-\mathbf{I}_{t} \cdot \Gamma_{\nabla_{t}}\right) \cdot \hat{W}_{y}\left(\mathbf{I}_{y} \cdot \Gamma_{\nabla_{t}}-W_{y t}\right)+W_{t t}\right]
\end{aligned}
$$

whose terms are explicitly defined and reported in [5].

One of the most important relations in the procedure is

$$
\tilde{\boldsymbol{\psi}}_{y}=\hat{W}_{y}\left(\mathbf{I}_{y} \cdot \Gamma_{\nabla_{t}}-W_{y t}\right) \cdot \tilde{\boldsymbol{\psi}}_{t}
$$

with $\tilde{\psi}_{y}=\left|0,0, \tilde{E}_{y}, 0,0, \tilde{H}_{y}\right|^{t}$ that relates the discontinuous longitudinal component to transverse components without a partial derivative along $y$ (only the third and the sixth rows are not null). 
Note that the transverse equations (4) are defined in the Euclidean space of dimension 4 instead of 6, since the third and sixth rows are null for the definition (3).

In the following we consider invariance of the geometry along $z$ as for stratified media. With this limitation, if the sources depend on an $e^{-j \alpha_{0} z}$ factor, the total field also depends on the same factor - that is, $\tilde{\boldsymbol{\psi}}_{c}(y, \boldsymbol{\rho})=\tilde{f}(y, x) e^{-j \alpha_{o} z}$. Furthermore, to study the variation of the fields in $x$, we introduce the Fourier transform

$$
f(y, \eta)=\int_{-\infty}^{\infty} \tilde{f}(y, x) e^{j \eta x} d x
$$

That yields from (4) the ordinary differential equations

$$
-\frac{d}{d y} \boldsymbol{\psi}_{t}(\eta, y)=M(\eta) \boldsymbol{\psi}_{t}(\eta, y)
$$

with $M(\eta)=\tilde{M}\left(-j \alpha_{o},-j \eta\right)$; see (5).

The definition of the explicit form of $M(\eta)$ and related properties need symbolic elaboration that can be performed with the help of software like Wolfram Mathematica [11]. In [5], $M(\eta)$ is reported for isotropic and anisotropic cases.

\section{Circuit Model of an Arbitrary Semi-Infinite Layer}

To introduce circuit modeling, we define as voltage and current the components in the Euclidean space of dimension 2 related to the Fourier transforms of $\tilde{\psi}_{t}$. More precisely, we define

$$
\psi_{t}(\eta, y)=|\mathbf{V}(\eta, y), \mathbf{I}(\eta, y)|^{t}
$$

with

$$
\begin{gathered}
\mathbf{V}(\eta, y)=\int_{-\infty}^{\infty}\left|\begin{array}{c}
\tilde{E}_{z}(x, y) \\
\tilde{E}_{x}(x, y)
\end{array}\right| e^{j \eta x} d x, \\
\mathbf{I}(\eta, y)=\int_{-\infty}^{\infty}\left|\begin{array}{c}
\tilde{H}_{z}(x, y) \\
\tilde{H}_{x}(x, y)
\end{array}\right| e^{j \eta x} d x
\end{gathered}
$$

We consider the plane $y=y_{o}$ and an arbitrary stratified medium located at $y>y_{o}$ in the presence of arbitrary sources. The linearity of the problem imposes that $\mathbf{V}$ $\left(\eta, y_{o}\right)$ and $\mathbf{I}\left(\eta, y_{o}\right)$ satisfy the equation

$$
\mathbf{V}=\mathbf{V}_{s}+Z_{e} \mathbf{I}
$$

where $\mathbf{V}=\mathbf{V}\left(\eta, y_{o}\right), \mathbf{I}=\mathbf{I}\left(\eta, y_{o}\right)$, and $\mathbf{V}_{s}=\mathbf{V}_{s}(\eta)$ and $Z_{e}=Z_{e}(\eta)$ are called the Thevenin voltage and the Thevenin impedance, respectively. We estimate the Thevenin voltage $\mathbf{V}_{s}$ at $y=y_{o}$ by imposing a perfect magnetically conducting boundary condition at $y=y_{o}-$ that is, $\mathbf{I}=0$. The impedance $Z_{e}$ is the $2 \times 2$ matrix that relates $\mathbf{V}$ and $\mathbf{I}$ when the sources are vanishing in the region $y>y_{o}$. The circuit model of the region $y>y_{o}$ is illustrated in Figure 1. The Norton representation is the

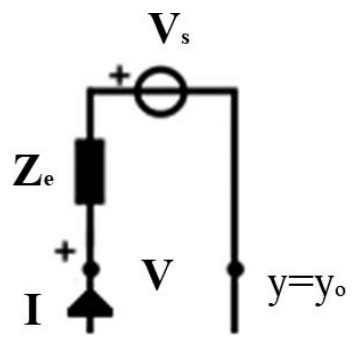

Figure 1. Thevenin representation of the half-space $y>y_{o}$.

dual circuit of the Thevenin one. Similar consideration apply in the stratified region located at $y<y_{o}$.

In the Thevenin model of a half-infinite layer, $Z_{e}$ $=Z_{e}(\eta)$ represents the characteristic impedance of the medium. In particular, the matrix $\vec{Z}_{c}=\vec{Z}_{c}(\eta)$ is the characteristic impedance that relates $\mathbf{V}$ and $\mathbf{I}$ in the direction $y>y_{o}$ in the absence of sources, and the matrix $\bar{Z}_{c}=\bar{Z}_{c}(\eta)$ relates $\mathbf{V}$ and $-\mathbf{I}$ in the direction $y<$ $y_{o}$.

\section{Circuit Model of an Arbitrary Multilayer Slab}

Let us consider a homogeneous slab defined between $y=0$ and $y=d$. The solution of (8) yields

$$
\begin{aligned}
\left|\begin{array}{c}
\mathbf{V}_{0} \\
\mathbf{I}_{0}
\end{array}\right| & =\mathcal{T}\left|\begin{array}{c}
\mathbf{V}_{d} \\
\mathbf{I}_{d}
\end{array}\right|, \quad \mathbf{V}_{0}=\left.\mathbf{V}\right|_{y=0}, \quad \mathrm{I}_{0}=\left.\mathrm{I}\right|_{y=0}, \\
\mathbf{V}_{d} & =\left.\mathbf{V}\right|_{y=d}, \quad \mathrm{I}_{d}=\left.\mathrm{I}\right|_{y=d}
\end{aligned}
$$

with the transmission matrix of the slab $0<y<d$ defined by

$$
\mathcal{T}=\mathcal{T}(\eta)=e^{M(\eta) d}=\left|\begin{array}{ll}
\mathbf{A}(\eta) & \mathbf{B}(\eta) \\
\mathbf{C}(\eta) & \mathbf{D}(\eta)
\end{array}\right|
$$

This $4 \times 4$ matrix has as elements the $2 \times 2$ matrices $\mathbf{A}$, $\mathbf{B}, \mathbf{C}, \mathbf{D}$ defined in terms of the $M$ matrix of dimension 4 (see section 2). Figure 2 shows a convenient representation of (12) in terms of a two-port network model. This representation is also valid for a slab constituted by a cascade of $s$ homogeneous consecutive slabs 1, 2, 3, ... In this case the transmission matrix of the multilayer slab is the product of the transmission matrices relevant to each slab:

$$
\mathcal{T}=\left|\begin{array}{ll}
\mathbf{A} & \mathbf{B} \\
\mathbf{C} & \mathbf{D}
\end{array}\right|=\mathcal{T}_{1} \mathcal{T}_{2} \mathcal{T}_{3} \ldots=e^{M_{1} d_{1}} e^{M_{2} d_{2}} e^{M_{3} d_{3}} \ldots
$$

\section{The Eigenvalues and the Eigenvectors}

The eigenvalues and eigenvectors of the matrix $M$ reported in (8) are very important in studying the solution of the equation. For example, they allow the evaluation functions of $M$ such as the exponentials that appear in (13) and (14). We study the eigenvalues and eigenvectors of $M$ defined in the Euclidean space of dimension 4 (see section 2): 

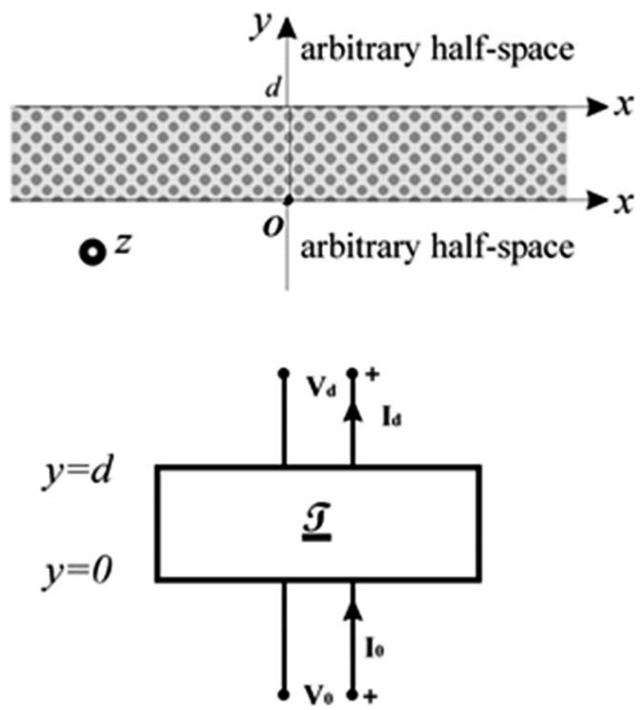

Figure 2. Top: Slab filled by an arbitrary linear medium. Bottom: Network equivalent model.

$$
M(\eta) \boldsymbol{\psi}^{(i)}(\eta)=\gamma_{i}(\eta) \boldsymbol{\psi}^{(i)}(\eta)
$$

where $\gamma_{i}(\eta)$ and $\psi^{(i)}(\eta)$ are, respectively, the eigenvalues and the eigenvectors. Since $M$ is a semisimple matrix, we have

$$
M=U J U^{-1}
$$

where $J=\operatorname{diag}\left[\gamma_{1}(\eta), \ldots, \gamma_{4}(\eta)\right], \quad U=\left[\boldsymbol{\psi}^{(1)}(\eta), \ldots\right.$, $\left.\boldsymbol{\psi}^{(4)}(\eta)\right]$, and $\boldsymbol{\psi}^{(i)}(\eta)=\left|\psi_{1}^{(i)}(\eta), \psi_{2}^{(i)}(\eta), \psi_{3}^{(i)}(\eta), \psi_{4}^{(i)}(\eta)\right|^{t}$.

In the presence of a passive medium, we observe that two eigenvalues (say, $\gamma_{1}, \gamma_{2}$ ) present a nonnegative real part and the other two, $\gamma_{3}, \gamma_{4}$, present a nonpositive real part, whence $\boldsymbol{\psi}^{(i)}(\eta)(i=1,2)$ are called progressive eigenvectors and $\psi^{(i)}(\eta)(i=3,4)$ are called regressive eigenvectors. The eigenvectors of $M$ provide the characteristic impedance of a medium. In fact [9], we have

$$
\begin{gathered}
\overrightarrow{\mathbf{Z}}_{c}=\left|\begin{array}{ll}
\psi_{1}^{(1)} & \psi_{1}^{(2)} \\
\psi_{2}^{(1)} & \psi_{2}^{(2)}
\end{array}\right| \cdot\left|\begin{array}{cc}
\psi_{3}^{(1)} & \psi_{3}^{(2)} \\
\psi_{4}^{(1)} & \psi_{4}^{(2)}
\end{array}\right|^{-1}, \\
\overrightarrow{\mathbf{Z}}_{c}=\left|\begin{array}{ll}
\psi_{1}^{(3)} & \psi_{1}^{(4)} \\
\psi_{2}^{(3)} & \psi_{2}^{(4)}
\end{array}\right| \cdot\left|\begin{array}{ll}
\psi_{3}^{(3)} & \psi_{3}^{(4)} \\
\psi_{4}^{(3)} & \psi_{4}^{(4)}
\end{array}\right|^{-1}
\end{gathered}
$$

Furthermore, the transmission matrix $e^{M(\eta) d}$ (13) is obtained by $e^{M(\eta) d}=U e^{J d} U^{-1}$, where $e^{J d}=$ $\operatorname{diag}\left[e^{\gamma_{1}(\eta) d}, \ldots, e^{\gamma_{4}(\eta) d}\right]$.

\section{Plane Waves in an Arbitrary Medium}

Plane waves in an arbitrary medium are solutions of the transverse equations (4) with (5) of the form

$$
\psi_{\mathbf{t}}=\psi_{\mathbf{0}} e^{-j \mathbf{k} \cdot \mathbf{r}}
$$

where $\mathbf{r}=|z, x, y|^{t}$ is the observation point, $\mathbf{k}=$ $\left|k_{y}, \alpha_{o}, \eta_{o}\right|^{t}$ is the propagation vector, and $\psi_{\mathrm{o}}$ is a constant vector of dimension 4. Taking into account that $\frac{\partial}{\partial y}=-j k_{y},(4)$ becomes

$$
j k_{y} \psi_{\mathbf{0}}=\mathrm{M}\left(\eta_{o}\right) \psi_{\mathbf{0}}
$$

where $k_{y}$ are related to the eigenvalues of matrix $M$ as defined in section 5. For a given set of $\alpha_{o}, \eta_{o}$, we have four possible propagation constants $k_{y}$ and four polarizations $\psi_{0}$ :

$$
j k_{y i}=\gamma_{i}(\eta), \quad \boldsymbol{\psi}_{\mathbf{o} i}=\boldsymbol{\psi}^{(i)}(\eta), \quad i=1,2,3,4
$$

We call the plane waves where $i=1,2$ progressive and the plane waves where $i=3,4$ regressive. Examples of values are given in [12]. For each value of $k_{y}$ (we omit the subscript $i$ ) we define a propagation vector and a propagation constant,

$$
k=|\mathbf{k}|=\sqrt{\alpha_{o}^{2}+\eta_{o}^{2}+k_{y}^{2}}, \quad \tau_{o}=\sqrt{k^{2}-\alpha_{o}^{2}}
$$

that identify the direction of the wave in terms of zenithal angle $\beta$ and azimuthal angle $\varphi_{o}$ : $\eta_{o}=-\tau_{o} \sin \varphi_{o}, \alpha_{o}=k \cos \beta$. We recall that the field $\psi_{t}$ contains only the transverse components of the electromagnetic field. The discontinuous components $E_{y}$ and $H_{y}$ are related to the transverse ones through (6).

\section{The Reflection Problem}

Considering the network modeling of a semiinfinite medium, we build the network representation of the reflection problem as shown in Figure 3.

In the network representation, the impedances $Z_{c 1}$ and $Z_{c 2}$ are the characteristic ones obtained in (17). We observe that the model is complete, considering sources in region $1(y>0)$ that yield (through imposing perfect magnetically conducting termination at $y=0$ ) the Thevenin voltage $2 \mathbf{V}^{\text {inc }}\left(\alpha_{o}, \eta_{o}\right)$, where $\mathbf{V}^{\text {inc }}\left(\alpha_{o}, \eta_{o}\right)$ is the incident voltage at $y=0$. The incident voltage $\mathbf{V}^{\text {inc }}$ $\left(\alpha_{o}, \eta_{o}\right)$ is related to the regressive incident plane wave that constitutes the source in region 1 . Without loss of generality, we suppose the presence of only one kind of incident plane: $\mathbf{V}^{\text {inc }}\left(\alpha_{o}, \eta_{o}\right)=\mathbf{V}^{\text {inc }}=V_{o} \mathbf{V}^{(i)}$, where $V_{o}$ is the intensity of the plane wave and $\mathbf{V}^{(i)}$ is the voltage part (that is, the first two components) of one of the regressive eigenvectors $\psi_{c}^{(i)}(i=3,4)$ of the matrix $M$ relevant to medium 1 -that is, $\left.\mathbf{V}^{(i)}=\psi_{1}^{(i)} \psi_{2}^{(i)}\right]$. Analyzing the circuit model of Figure 3, we have

$$
\begin{aligned}
& \mathbf{I}=-\left(Z_{c 1}+Z_{c 2}\right)^{-1} 2 \mathbf{V}^{\text {inc }} \\
& \mathbf{V}=-Z_{c 2} \mathbf{I}=T \mathbf{V}^{\text {inc }}=\mathbf{V}^{\text {inc }}+\Gamma \mathbf{V}^{\text {inc }}=\mathbf{V}^{\text {inc }}+\mathbf{V}^{\text {ref }}
\end{aligned}
$$

where $\mathbf{V}^{\text {ref }}(0)=\mathbf{V}^{\text {ref }}=\Gamma \mathbf{V}^{\text {inc }}=\Gamma \mathbf{V}^{\text {inc }}(0)$ defines the reflected voltage at $y=0$ and the $2 \times 2$ reflection matrix $\Gamma$ is given by

$$
\begin{aligned}
\Gamma & =T-1_{3}=2 Z_{c 2}\left(Z_{c 1}+Z_{c 2}\right)^{-1} \\
& =\left(Z_{c 2}+Z_{c 1}\right)\left(Z_{c 1}+Z_{c 2}\right)^{-1}
\end{aligned}
$$

When the structure is excited or illuminated with one 

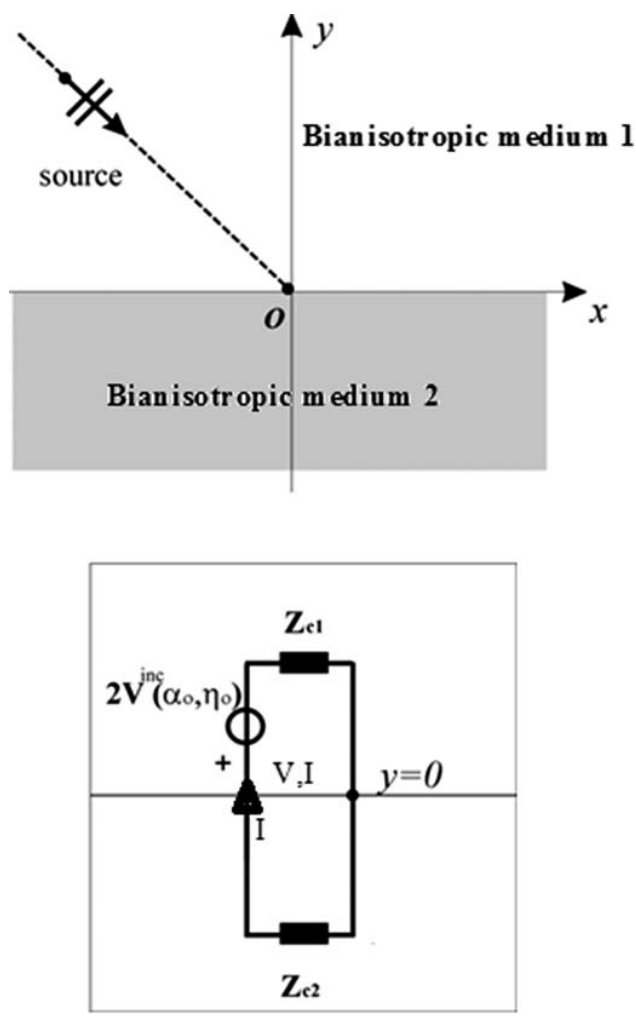

Figure 3. Top: Plane wave reflection between two semi-infinite homogenous bianisotropic media. Bottom: Network modeling based on the Thevenin's equivalence.

regressive wave (either $i=3$ or $i=4$ ), in general we get as reflected waves all the progressive reflected waves $(i=1,2)$ of medium 1 and all the regressive transmitted waves $(i=3,4)$ of medium 2 . We can evaluate the coupling coefficient in terms of the reflection matrix $\Gamma$. Taking into account that the reflected wave

$$
\mathbf{V}^{\mathrm{ref}}(y)=C_{o 1} e^{-\gamma_{1} y} \mathbf{V}^{(1)}+C_{o 2} e^{-\gamma_{2} y} \mathbf{V}^{(2)}
$$

contains all the progressive plane waves present at $y>$ 0 , we obtain the excitation coefficients $C_{o 1}, C_{o 2}$ by considering that at $y=0$ we get

$$
\begin{aligned}
\mathbf{V}^{\text {ref }}(0) & =\Gamma \mathbf{V}^{\text {inc }}(0)=C_{o 1} \mathbf{V}^{(1)}+C_{o 2} \mathbf{V}^{(2)} \\
& =\left|\begin{array}{ll}
\psi_{1}^{(1)} & \psi_{1}^{(2)} \\
\psi_{2}^{(1)} & \psi_{2}^{(2)}
\end{array}\right|\left|\begin{array}{l}
C_{o 1} \\
C_{o 2}
\end{array}\right|
\end{aligned}
$$

which yields

$$
\begin{aligned}
\left|\begin{array}{l}
C_{o 1} \\
C_{o 2}
\end{array}\right| & =\left|\begin{array}{ll}
\psi_{1}^{(1)} & \psi_{1}^{(2)} \\
\psi_{2}^{(1)} & \psi_{2}^{(2)}
\end{array}\right|^{-1} \mathbf{V}^{\mathrm{ref}}(0) \\
& =\left|\begin{array}{ll}
\psi_{1}^{(1)} & \psi_{1}^{(2)} \\
\psi_{2}^{(1)} & \psi_{2}^{(2)}
\end{array}\right|^{-1} \Gamma \mathbf{V}^{\text {inc }}(0)
\end{aligned}
$$

Similar considerations apply for the evaluation of plane waves transmitted in medium $2(y<0)$. By indicating with $\gamma_{t i}$ and $\mathbf{V}_{t}^{(i)}(i=3,4)$ the regressive eigenvalues and eigenvectors of the matrix $\mathrm{M}_{\mathrm{t}}\left(\eta_{o}\right)$ defined in medium 2, for $y<0$ we have

$$
\begin{array}{r}
\mathbf{V}(y)=C_{t o 3} e^{-\gamma_{t 3} y} \mathbf{V}_{t}^{(3)}+C_{t o 4} e^{-\gamma_{t 4} y} \mathbf{V}_{t}^{(4)} \\
\left|\begin{array}{l}
C_{t o 3} \\
C_{t o 4}
\end{array}\right|=\left|\begin{array}{ll}
\psi_{t 1}^{(3)} & \psi_{t 1}^{(4)} \\
\psi_{t 2}^{(3)} & \psi_{t 2}^{(4)}
\end{array}\right|^{-1} T \mathbf{V}^{\text {inc }}(0)
\end{array}
$$

\section{Conclusions}

This work proposes a new effective method to estimate the contributions of geometrical optics in arbitrary linear stratified planar structures, based on equivalent network models and Bresler-Marcuvitz transversalization theory. The method is particularly useful for starting the analysis of novel complex canonical problems constituted of angular or stratified structures with the generalized Wiener-Hopf technique, as in [13].

\section{References}

1. V. G. Daniele and G. Lombardi, Scattering and Diffraction by Wedges 1, Hoboken, NJ, Wiley-ISTE, 2020.

2. V. G. Daniele and G. Lombardi, Scattering and Diffraction by Wedges 2, Hoboken, NJ, Wiley-ISTE, 2020.

3. V. G. Daniele, The Wiener-Hopf Technique for Wedge Problems, Internal Rep. ELT-2004-2, 255 DET, Politecnico di Torino, 2004.

4. V. G. Daniele and G. Lombardi, "The Wiener-Hopf Technique for Impenetrable Wedge Problems," International Conference Days on Diffraction, Saint Petersburg, Russia, June 28-July 1, 2005, pp. 50-61.

5. V. G. Daniele and G. Lombardi, "The Generalized Wiener-Hopf Equations for Wave Motion in Angular Regions: Electromagnetic Application," Proceedings of the Royal Society A, 477, 2252, August 2021, p. 20210040, doi: 10.1098/rspa.2021.0040.

6. V. G. Daniele and G. Lombardi, "The Generalized Wiener-Hopf Equations for the Elastic Wave Motion in Angular Regions," Proceedings of the Royal Society A, 4782021062420210624, 2022, http://doi.org/10.1098/ rspa.2021.0624, pp. 1-29.

7. V. G. Daniele, G. Lombardi, and R. S. Zich, "Radiation and Scattering of an Arbitrarily Flanged DielectricLoaded Waveguide," IEEE Transactions on Antennas and Propagation, 67, 12, December 2019, pp. 75697584.

8. V. G. Daniele and G. Lombardi, "Fredholm Factorization of Wiener-Hopf Scalar and Matrix Kernels," Radio Science, 42, 6, December 2007, p. RS6S01.

9. V. G. Daniele and R.S. Zich, The Wiener-Hopf Method in Electromagnetics, Edison, NJ, Scitech Publishing, 2014.

10. A. D. Bresler and N. Marcuvitz, Operator Methods in Electromagnetic Field Theory, Report R-495,56, PIB425, MRI Polytechnic Institute of Brooklyn, 1956.

11. Author, Mathematica, version NNN, Place of Publication, Wolfram Research, Inc., Mathematica, Version 12.3, Champaign, IL, 2021.

12. V. Daniele and R. D. Graglia, "Diffraction by an 
Imperfect Half Plane in a Bianisotropic Medium," Radio Science, 42, 6, December 2007, p. RS6S05, doi: 10.1029/ 2007RS003674.

13. V. Daniele and G. Lombardi, "Wiener-Hopf Method for Arbitrary Linear Scattering Problems in Angular Re- gions," XXXIV General Assembly and Scientific Symposium (GASS) of the International Union of Radio Science (Union Radio Scientifique Internationale-URSI), Rome, Italy, August 28-September 4, 2021. 